\title{
Case-Based Assessment of Medical Students’ Knowledge and Skill on Principles of Drug Prescription
}

\author{
Leila Bazrafkan, \\ Education Development and Research Center \\ Shiraz University of Medical Sciences, Shiraz, Iran \\ Tel: 98-711-233-3064Ｅ-mail: Iran.bazrafkanl@sums.ac.ir \\ Mohanmadreza Dehghani, \\ Education Development and Research Center \\ Shiraz University of Medical Sciences, Shiraz, Iran \\ Tel: 98-711-233-3064Ｅ-mail: Iran.dehghani@suma.ac.ir \\ Nasrin Shokrpour (Corresponding Author) \\ English Department, Faculty of Paramedical Sciences \\ Shiraz University of Medical Sciences, Shiraz, Iran \\ Tel: 98-711-228-9113Ｅ-mail: shokrpourn@gmail.com
}

\begin{abstract}
Ali Hekmatpanah Dehkor,
School of Medicine, Shiraz University of Medical Sciences, Shiraz, Iran

Tel: 98-711-233-3064Ｅ-mail: bazrafcan@yahoo.com
\end{abstract}

Received: Nov. 24, 2012 Accepted: December 10, 2012 Published: February 1, 2013

doi:10.5296/jse.v3i1.2755 ～URL: http://dx.doi.org/10.5296/jse.v3i1.2755

\begin{abstract}
Prescription of drugs follows some principles about which the medical students should be aware, and they should practice them efficiently so that they become experienced in the field. This study aims to determine the practice and knowledge of drug prescription in senior medical students. A cross-sectional study was conducted on a sample of 140 students from
\end{abstract}


Shiraz University of Medical Sciences. Data were collected using self administered questionnaires in the format of case study and essay questions. The questions were designed based on principles of drug prescription presented to the interns in Shiraz University of Medical Sciences on the subjects including drug indication, interaction and side effects; contraindication of drugs in pregnancy; maximum drugs prescribed in one prescription letter; and the rules of insurance organizations in Iran. The data analysis was performed using SPSS, version 15. Content validity of multiple choice questions was confirmed by expert opinion and the consensus of four reference texts. 120 students participated in this study with a response rate of $85.7 \%$. As to the knowledge of medical students, onlly $34.18 \%$ of them were competent in prescribing drugs; however, no one got a full score in the exam. $27.73 \%$ of them answered the questions incompletely, and 30.09\% of them showed wrong prescription. Most mistakes were related to drug interaction, antibiotics and analgesics drugs (68.3\%). As to the reference books of pharmacology, $16.9 \%$ had complete knowledge, 54.8\% moderate level of knowledge, $28.3 \%$ had no information about that. This study shows that the knowledge of medical students as to drug prescription is inadequate. Also, many studies have approved that effective education has a significant effect on improvement of drug prescription by physicians. We recommend interventions such as curriculum development to increase the students' skills. This should be taken into account in further research and by public health professionals and health policy makers.

Keywords: Drug prescription, Knowledge, skills, Undergraduate medical students 


\section{Introduction}

Drug therapy is one of the most common approaches to treatment of diseases in medicine. (Babor et al., 2010; Malone et al., 2005; Laing, Hogerzeil, \& Ross-Degnan, 2001) Proper prescription and reasonable administration of drugs are the essential factors in the health of a community. (Sitanshu, Himanshu, \& Guru, 2010; Riedel \& Casillas, 2003) Drug administration has increased during the last decade in our country, leading to problems such as shortage or lack of some drugs. (Mohagheghi et al., 2005) For instance, inappropriate use of antibiotics gives rise to microbial resistance and an increase in the expenses for the patients and health care systems in our country. (Shekholeslami \& Asefzade, 1999) Drug interaction is another problem arising from careless drug prescriptions. This occurs in the body when two or more drugs are used by the patient simultaneously, leading to changes in the effect or toxicity of the drug - the fact that is emphasized in many studies. (Glintborg, Anderson, \& Dalhoff, 2005; Saverno, Malone, \& Kurowsky, 2009; Lafata, et al., 2006; Trujillo, 2009)

Drugs' side effect is an important issue in medical education As reported in many research and by food and drug deputy in the Ministry of Health in Iran, 8\% of the hospital admissions are due to drugs' side effects. (Rashidi \& Senobar Tahai, 2005) In a survey, 4000 prescriptions by 52 GPs practicing in Babol in 1378 were surveyed. In $30.4 \%$ of the prescriptions by GPs, there were differences in the drugs prescribed. So, most of the above-mentioned problems were observed in GPs' prescriptions, leading to an undesirable condition for the patients. (Moghaddammi, Ahedpasha, Mirbolooki, \& Baradaran Aghili, 1999)

Moreover, in a study conducted in Shiraz University of Medical Sciences aiming at a survey of the drug prescription pattern of GPs and determination of the effect of education on the approach to the prescription of drugs, it was found that the most common drugs prescribed were injections (58.8\%) and antibiotics (47.3\%). After educational interventions, the pattern of prescribing drugs improved significantly. Also, there was a significant difference in the prescription of injection drugs and non-steroid anti-inflammation drugs. (Zare, Razmjoo, Ghaeminia, Zeighami \& Aghamaleki, 2007)

There is evidence that many medical schools have tried to teach and organize pharmacology courses but a common problem in many medical schools is that undergraduate medical students learn little about drug prescription.(Cheraghali, Panahi, \& Alidadi, 2003; Azmi, Kay \& Kong, 2008; Vollebregt, et al., 2006; de Vries et al., 1995) In our country, the pharmacology course is offered in the physiopathology period while the students are loaded with subjects to be learned. Afterwards, when the clinical period starts, they have nothing to do with drugs and prescription. In the internship period, they are suddenly expected to prescribe drugs while they have forgotten most of what they have learned about it. (Panjeshahin \& Sabani Firoozi, 2004; Brunton, Lazo, \& Parker, 2006) Therefore, due to the importance of the medical students' information in drug prescription in the health systems and community health, this study was conducted. Therefore, there was an attempt in this study to survey the medical students' knowledge and skills in drug prescription immediately before their graduation. 


\section{Material and Methods}

A cross-sectional study was conducted on a sample of 140 students from Shiraz University of Medical Sciences. Their mean age was 24 years (SD_+1.74) (45\% female and 55\% male). Ethics requirements were ensured and data were collected using self administered questionnaires with the format of case study and essay questions. The questions were designed based on the principles of drug prescription presented to the interns of Shiraz University of Medical Sciences on the subjects including drug indication, interaction, and side effects; contraindication in use of drugs during pregnancy; maximum drugs prescribed in one prescription; and insurance organizations rules in Iran. The questionnaire consisted of two sections, the first part including questions on the students' demographic data such as age, sex, the year of entrance to the university, and their scores in the pre-internship exam. The second part consisted of questions on the students' level of knowledge about the principles of prescribing drugs in the form of case and MCQ as well as questions in which the student has to prescribe drugs for an unreal patient.

In each case, a simulated patient was presented to the students followed by some questions about the principles of drugs prescription. To analyze the data, the questions were divided into those on the knowledge about how to prescribe drugs, those on the appropriate form of prescription, and those on the rules governing prescription of drugs.

In the first case, the students were asked to observe the principles of drug interaction in a case. The second question was about the drug contra-indication during pregnancy. They were asked to determine which drugs should not be prescribed for a pregnant woman. The other questions were about drugs' side effects. To assess the student's knowledge about the proper format of prescription, the principles of prescribing medicine based on reference books (Goolman, 2006; Solberg et al., 2004) were categorized. Then, some questions were asked about the number of drugs prescribed in one prescription, drug dosage, duration of use, and the patient's medical information.

Data from the questionnaires filled out completely were coded and entered into SPSS, version 15. To summarize the data, we used descriptive statistics to find out the frequency, distributions and mean. The students were then asked what they did if they made a mistake when prescribing and then had to correct it. They were asked to prescribe medicine for a patient considering the following factors: proper prescription of the drug with its does, type, required amount, instruction for the use of drug for the mentioned patient, and the eligibility of the prescription, i.e. the date on the top of the prescription and signature of the physician. Each part had a specific score. In the section on knowledge about the rules of prescription, the price of available drugs, drugs under the insurance, and the level of knowledge about the drugs' reference books were the questions asked in different sections. 190 points were considered for the questions in the questionnaire in 9 sections, 20 for each question. The score 20 was allocated to each correct answer and zero to wrong ones. Therefore, there was a minimum score of zero, and a maximum score of 2 in each case. So, the minimum scores given to the students were zero and maximum 190 in this case-based exam. Due to experts judgments, the standard of performance for students' score in knowledge of drug prescription 
in 9 cases was determined as score (16-20) desirable, score (12-16) moderately desirable and score (>12) undesirable or poor performance.

\section{Results}

104 students participated in this study with a response rate of $86.7 \%$. The results revealed that the maximum score was 125 and minimum score 79.5 in this case-based exam. As to the knowledge of medical students, only $34.18 \%$ of them were competent in prescribing drugs; however, no one got a perfect score in the exam. $27.73 \%$ of them answered the questions incompletely, and $30.09 \%$ of them showed wrong prescription. Most mistakes were related to drag interaction, antibiotics and analgesic drugs (68.3\%). As to the reference books of pharmacology, $16.9 \%$ had complete knowledge, 54.8\% moderate level of knowledge, $28.3 \%$ had no information about that. Scores of drug interactions case were compared with those of pre-internship exam; the result was statistically significant. $(\mathrm{p}<.0005)$

Table 1 shows the total scores of girls and boys in the case-based exam. The data in Table 2 are based on the average standard for good, desirable, moderately desirable, and undesirable performance in the 9 items. Also, Table 3 shows average, variance and significant differences in the scores of girls and boys in separate domains in 9 items.

Table 1. Mean score and standard deviation among the males and females in total

\begin{tabular}{|c|c|c|c|}
\hline Group & N. & Min score & Total Test score \\
\hline male & 55 & $95.12 \pm 15.65$ & 180 \\
\hline female & 45 & $103.34+21.66$ & 180 \\
\hline
\end{tabular}

Table 2. Percentage and frequency of students' performance in knowledge of drug prescription

\begin{tabular}{|l|l|l|l|}
\hline Cases & $\begin{array}{l}\text { Undesirable } \\
\text { Poor }(>12)\end{array}$ & $\begin{array}{l}\text { Desirable } \\
\text { Medium (12-16) }\end{array}$ & $\begin{array}{l}\text { Desirable } \\
(16-20)\end{array}$ \\
\hline Drug interactions & $46(44.23)$ & $40(38.46)$ & $18(17.31)$ \\
\hline Drug prescription in pregnancy & $39(37.51)$ & $9(8.65)$ & $56(53.85)$ \\
\hline Maximum drug per prescription & $21(20.19)$ & - & $83(79.81)$ \\
\hline Drugs cost & $69(66.34)$ & - & $35(33.66)$ \\
\hline Drugs insurance & $80(76.92)$ & $22(21.15)$ & $2(1.92)$ \\
\hline Drugs side effects & $35(33.66)$ & $4(3.85)$ & $65(62.5)$ \\
\hline Correct mistake in prescription & $62(59.62)$ & - & $42(40.38)$ \\
\hline Correct format in prescribing & $9(8.65)$ & $67(64.42)$ & $28(26.92)$ \\
\hline Knowledge about reference book & $57(54.81)$ & $28(26.92)$ & $19(18.27)$ \\
\hline
\end{tabular}




\section{Macrothink}

Table 3. Mean score, standard deviation and p. value

\begin{tabular}{|c|c|c|c|c|c|}
\hline $\mathbf{N}$ & Topic & Group & $\mathbf{N}$ & Mean Score & P Value \\
\hline \multirow{2}{*}{1} & \multirow{2}{*}{ Drug interaction } & male & 55 & $12.12+4.65$ & \multirow{2}{*}{.04} \\
\hline & & female & 45 & $12.43+5.38$ & \\
\hline \multirow{2}{*}{2} & \multirow{2}{*}{ Drug prescription in pregnancy } & male & 55 & $14.56 \pm 6.44$ & \multirow{2}{*}{.978} \\
\hline & & female & 45 & $14.50 \pm 5.55$ & \\
\hline \multirow{2}{*}{3} & \multirow{2}{*}{ Maximum drug per } & male & 55 & $16.97 \pm 7.24$ & \multirow{2}{*}{.05} \\
\hline & & female & 45 & $15.10+5.82$ & \\
\hline \multirow{2}{*}{4} & \multirow{2}{*}{ Drugs cost } & male & 55 & $14.92+4.67$ & \multirow{2}{*}{.191} \\
\hline & & female & 45 & $14.49+4.23$ & \\
\hline \multirow{2}{*}{5} & \multirow{2}{*}{ Drugs insurance } & male & 55 & $13.10 \pm 6.31$ & \multirow{2}{*}{.03} \\
\hline & & female & 45 & $14.20 \pm 5.92$ & \\
\hline \multirow{2}{*}{6} & \multirow{2}{*}{ Drugs side effects } & male & 55 & $13.80 \pm 5.63$ & \multirow{2}{*}{.04} \\
\hline & & female & 45 & $14.10 \pm 5.32$ & \\
\hline \multirow{2}{*}{7} & \multirow{2}{*}{ Correct mistake in prescription } & male & 55 & $14.80 \pm 4.55$ & \multirow{2}{*}{.549} \\
\hline & & female & 45 & $13.10 \pm 5.75$ & \\
\hline \multirow{2}{*}{8} & \multirow{2}{*}{ Correct format in prescribing } & male & 55 & $15.04 \pm 4.43$ & \multirow{2}{*}{.679} \\
\hline & & female & 45 & $16.83 \pm 3.65$ & \\
\hline \multirow{2}{*}{9} & \multirow{2}{*}{ Knowledge about reference book } & male & 55 & $17.60 \pm 3.14$ & \multirow{2}{*}{.329} \\
\hline & & female & 45 & $17.24 \pm 3.89$ & \\
\hline
\end{tabular}

Table 1 shows male and female students’ total exam score

\section{Discussion and conclusion}

The objective of the present study was to assess the senior medical student's knowledge about the principles of drug prescription. According to the results, it seems that the majority of medical students do not have sufficient knowledge about prescriptions in three domains of knowledge, proper format, and the rules of prescribing medicine. There was no significant difference among the 9 domains. These results are in the same line with those of Moghaddam, et al.'s study (1999) performed in Babol on the prescriptions given by general practitioners, revealing the undesirable status of drug prescription by these physicians.

According to our results, the most problems medical students are faced with are related to drugs' side effects. Drugs' interaction and side effects are one of the current significant subjects under discussion. Knowledge about the new treatment protocols is only one part of the logical treatment; however, interference with the prescribed drugs or with those prescribed earlier is more important. This should be further taken into account by physicians since drugs' side effects might add to the patient's previous problems. (Chambliss et al., 2012; Mohagheghi et al., 2005; Durrence, Dipiro, \& May, 1985; Berwick \& Finkelstein, 2010; WHO, 2009) Also, most of the students did not know the rules governing; the prescription of drugs. These results accord with those of a study performed on GPs in Shiraz, indicating a significant reduction in considering drugs' interference after instructions. As to drug prescription, $53.8 \%$ were not adequately aware of the drugs indicated in pregnancy and $46.2 \%$ were fairly aware of them. (Zare et al., 2007) 
Based on the results, it is necessary to mention that there was a significant difference in considering drug interaction in question no.1. $(\mathrm{p}=0.01)$. It can be concluded that those students with higher grades in pre-internship exam had more knowledge about prescribing drugs. However, based on the students' scores in pre-internship exam in the proper format of prescription, there was no significant difference $(\mathrm{P}>0.05)$. This can be due to the fact that prescribing drugs is a practical task and the core of medical students' curriculum, so there is a need for its frequent practice in different phases of the program as in Dandy spiral curriculum. (Ellis, Goodyear, Brillant \& Prosser, 2008, Harden, 1999; Orme \& Reidenberg, 1989)

Moreover, in the current study the number of the drugs prescribed was 4.44 which is very similar to the results of the study conducted in Babol with a mean of 4.48 drugs prescribed in the insurance book. (Zare et al., 2007) In a study on 12 developing countries, this mean was 2.2, more than half of which being less than1.5.(Laing et al., 2001) The culture of drug administration among the public will surely not change without a change in the physicians' duties. Therefore, to reach this objective, there is a need for more cooperation between the physicians and pharmacists. Education of medical students and physicians on the logical drug prescription should be taken more seriously and followed by continual medical education centers in the country. (Orme, et al., 1990; Han \& Maxwell, 2006; Nierenberg, 1991)

In this study, $50 \%$ of the students have mentioned that they had learned to prescribe drugs from the senior students and residents, while in Australia 92\% of the medical students believed so. (Coombess, Mitchell \& Stowasser, 2007) Since $39.4 \%$ of the students in the present study mentioned that they have learned how to prescribe medicine haphazardly without any designed program or course, the main reason for this reduction can be lack of attention and surveillance about this significant issue. Moreover, most of the participants of this study did not consider the education offered by the medical school sufficient. Similarly, more than $90 \%$ of the students under the study conducted in Australia believed so. According to the results of that study, Australian medical students need more effective and better education as to drugs and their prescription with generic names. (Coombesi, et al., 2007)

As to familiarity with the drugs under insurance, none of the students had sufficient knowledge and $21.2 \%$ of them had a moderate level of knowledge about it. With regard to the results, it seems necessary to pay more attention to continuous and effective education of medical experts on these rules to protect the patient from any loss resulting from insurance rules and regulations in a clinical pharmacology course. (Weingart, et al., 2009) As shown by a survey conducted under the sponsorship of the WHO in European medical schools, an average of only $28 \mathrm{~h}$ was allocated to teaching clinical pharmacology. (Orme et al., 1990)

\section{Recommendations}

The subjects participating in this study provided the researcher with their views in the last part of the questionnaire. According to them, the time of presenting the pharmacology course is not suitable. They recommend that this course should be presented with more units and useful course contents in the middle of the clinical period, especially before internship period. It is also suggested that repeated courses on how to prescribe medicine in regular intervals are highly influential. The use of interactive and creative techniques, such as simultaneous 
interactive lecturing, and small group sections in pharmacology courses, facilitates the application of similar learning methods in the following academic years by the students themselves, because students have already learned and experienced the active learning methods. The use of pedagogical methods and staff development activities can help teachers to improve their instructional skills. (Harden, 1999; Orme \& Reidenberg, 1989; Han \& Maxwell, 2006; Orme et al., 1990) However, there is an urgent need to revise the curriculum, modify educational objectives and hold new courses.

\section{Acknowledgement}

The authors would like to thank Dr. Kojoori, the director of EDC in SUMS, Dr. Shams, Dr Nejabat and all those who cooperated in different parts of this study.

\section{References}

Azmi, M., Kay, S., A, \& Kong, C.M. (2008). A national survey on knowledge and perceptions of senior medical students in Australia about generic medicines. MJA, 188(2), 123-124.

Babor, T., Caulkins, J., Edwards, G., Fischer, B., Foxcroft, D., \& Humphreys, K., et al. (2010). Drug policy and the public good. Oxford: Oxford University Press.

Berwick, D., \& Finkelstein, J. (2010). Preparing medical students for the continual improvement of health and health care: Abraham Flexner and the new "'Public Interest"'. Acad Med, 85, 56-65. http://dx.doi.org/10.1097/ACM.0b013e3181ead779

Brunton, L., Lazo, J., \& Parker, K. (2006). Goodman and Gilman's the pharmacological basis of therapeutics, $11^{\text {th }}$ edition. University of California publications.

Chambliss, W.G., Carroll W.A., Kennedy, D., Levine, D., Moné, M.A., Ried L.D., Shepherd, M., \& Yelvigi, M. (2012). Role of the pharmacist in preventing distribution of counterfeit medications. J Am Pharm Assoc., 52, 195-199. http://dx.doi.org/10.1331/JAPhA.2012.11085

Cheraghali, A., Panahi, U., \& Alidadi, A. (2003). A survey of the prescriptions from the hospitals of one of the universities of medical sciences in Tehran. Medicine and Purification, 44, 30-36.

Coombes, I., Mitchell, C., \& Stowasser, D. (2007). Safe medication practice tutorials: a practical approach to preparing prescribers. Clin Teach, 4, 128-34. http://dx.doi.org/10.1111/j.1743-498X.2007.00164.x

de Vries, T. P., Henning, R. H., Hogerzeil, H. V., Bapna, J..S., Bero, L., Kafle, K..K., Mabadeje, A., Santoso, B., \& Smith, A. J. (1995). Impact of a short course in pharmacotherapy for undergraduate medical students: an international randomized controlled study. Lancet, 346(8988), 1454-1457. http://dx.doi.org/10.1016/S0140-6736(95)92472-8

Durrence, C. W., Dipiro, J. T., \& May, J. R. (1985). Potential drug interactions in surgical patients. Am J Hosp Pham, 42, 1553-1555. 


\section{$\triangle$ Macrothink}

Journal of Studies in Education

ISSN 2162-6952

2013, Vol. 3, No. 1

Ellis, R. A., Goodyear, P., Brillant, M., \& Prosser, M. (2008). Student experiences of PBL in pharmacy: Face-to-face and on-line approaches. Advances in Health Siciences Education, 13(5), 675-687. http://dx.doi.org/10.1007/s10459-007-9073-3

Glintborg, B., Anderson, S.E. \&, Dalhoff, K. (2005). Drug-drug interactions among recently hospitalised patients- frequent but mostly clinically insignificant. Eur J Clin Pharmacol, 61(9), 675-681.

Han, W.H., \& Maxwell, S.R. (2006). Are medical students adequately trained to prescribe at the point of graduation? Views of first year foundation doctors. Scott Med J., 51(4), 27-32 http://dx.doi.org/10.1258/rsmsmj.51.4.27

Harden, R. M. (1999). What is a spiral curriculum? Med teach, 21(2), 141-143. http://dx.doi.org/10.1080/01421599979752

Lafata, J. E., Schultz, L., Simpkins J. et al (2006). Potential drug-drug interactions in the $\begin{array}{lllll}\text { outpatient setting. } & \text { Med } & \text { 534-541. }\end{array}$ http://dx.doi.org/10.1097/01.mlr.0000215807.91798.25

Laing, R.O., Hogerzeil, H.V., \& Ross-Degnan, D. (2001). Ten recommendations to improve use of medicine in developing countries. Health Policy and Planning, 16, 13-20. http://dx.doi.org/10.1093/heapol/16.1.13

Malone, D.C., Hutchins, D.S., Haupert, H. et al. (2005). Assessment of potential drug-drug interactions with a prescription claims database. Am J Health-Syst Pharm, 62(19), 1983-1991. http://dx.doi.org/10.2146/ajhp040567

Moghaddammi, A., Aahedpasha, Y., Mirbolooky, M.R., \& Baradaran Aghili, M. (1999). Prescription indices as to drug prescription patterns used by general practitioners is Babol. Babol University of Medical Sciences Journal, 3, 21-26. http://dx.doi.org/10.1002/pds.1057

Mohaheghi, M.A., Mosavi-Jarrahi, A., Khatemi Moghaddam, M., Afhami, S., Khodai, S., \& Azemoodeh, O. (2005). Community based outpatient practice of antibiotics use. Tehran. Pharmacoepidemiol Drug Saf., 14, 135-138

Nierenberg, D.W. (1991). A core curriculum for medical-students in clinical-pharmacology and therapeutics. J Clin Pharmacol, 31(4), 307-311.

Orme, M., Reidenberg, M. (1989). The teaching of clinical-pharmacology in Europe and $\begin{array}{lllll}\text { North-America. } & \text { Trends } & \text { Pharmacol } & \text { Sci., } & 10(6),\end{array}$ http://dx.doi.org/10.1016/0165-6147(89)90265-4

Orme, M., Sjoqvist, F., Bircher, J., Bogaert, M., Dukes, M.N., Eichelbaum, M., Gram, L.F., Huller, H., Lunde, I., \& Tognoni, G. (1990). WHO Working Group on Clinical Pharmacology.The teaching and organisation of clinical pharmacology in European medical schools. Eur J Clin Pharmacol, 38(2), 101-105.

Panjeshahin, M.R., \& Sabani Firoozi, M. (2004). A guide to proper prescribing. Shiraz University of Medical Sciences publications. Shiraz, Iran. 4th edition 


\section{Macrothink Institute ${ }^{\mathrm{TM}}$}

Rashidi, K., \& Senobar Tahai, S. N. (2005). A survey of the drug interaction as observed in the prescriptions of Kordestan province physicians under insurance in 2000. Kordestan University of Medical Sciences J., 10, 78-84.

Riedel, M.A., \& Casillas AM. (2003). Adverse drug reaction: types and treatment options. American family physician, 68, 1781-1790.

Saverno, K.R., Malone, D.C., \& Kurowsky, J. (2009). Pharmacy Students' Ability to identify potential drug-drug interactions. Am J Pharm Educ., 73(2), Article 27. http://dx.doi.org/10.5688/aj730227

Sheikholeslami,, H., \& Asefzade, S. (1999). Antibiotics in the prescription of physicians in Ghazvin. Gilan University of Medical Sciences Journal, 8(31-320), 35-41.

Sitanshu, S.K, Himanshu, S.P, \& Guru P.M.. (2010). Concept of Essential Medicines and Rational Use in Public Health. Indian $J$ Community Med., 35(1), 10-13. http://dx.doi.org/10.4103/0970-0218.62546

Solberg, L.I., Hurley, J.S., \& Roberts, M.H., et al. (2004). Measuring patient safety in ambulatory care: potential for identifying medical group drug-drug interaction rates using claims data. Am J Manag Care, 10(11), 753-759Gh

Trujillo, J.A. (2009). Drug interactions elective course. Am J Pharm Educ., 73(4), Article 72. http://dx.doi.org/10.5688/aj730472

Vollebregt, J.A., van Oldenrijk, J., Kox, D., van Galen, S.R., Sturm, B., Metz, J.C., Richir, M.C., de Haan, M., Hugtenburg, J.G., \& de Vries, T.P. (2006). Evaluation of a pharmacotherapy context-learning programme for preclinical medical s.tudents. $\mathrm{Br} \mathrm{J}$ Clin Pharmacol, 62(6), 666-672. http://dx.doi.org/10.1111/j.1365-2125.2006.02742.x

Weingart, S.N., Simchowitz, B., Padolsky, H., Isaac, T., Seger, AC, Massagli, M., et al. (2009). An empirical model to estimate the potential impact of medication safety alerts on patient safety, health care utilization, and cost in ambulatory care. Arch Intern Med., 169(16), 1465-1467. http://dx.doi.org/10.1001/archinternmed.2009.252

WHO. (2009). Patient safety curriculum guide for medical schools. World Alliance for Patient, Safety World Health Organisation, Geneva:WHO Press.

Zare, N., Razmjoo, M., Ghaeminia, M., Zeighami, B., \& Aghamaleki, Z. (2007). A survey of the effect of education on the methods of prescribing medicine by GPS under insurance in Shiraz. The East Hesitation, 9(4), 255-261. 